\title{
A Study on Architectural Plans for Modern Industrial Heritage and Urban Space Revitalization - Architectural Plan Based on the Concept of Restoration and Interact Boundary
}

\author{
Yujin Lee ${ }^{1}$, Donghyeog Choi $^{2}$ \\ ${ }^{1}$ Undergraduate Student, Dept. of Architecture, Gachon University, Korea, febten98@naver.com \\ ${ }^{2}$ Associate Professor, Dept. of Architecture, Gachon University, Korea, donghyeog@gachon.ac.kr \\ Corresponding author: Donghyeog Choi
}

\begin{abstract}
In order to preserve the historical and cultural values of the modern industrial heritage and to find a way to interact with the modern industrial heritage, which has been cut off from the city, this study proposes a plan for urban regeneration as an alternative to restore the modern industrial heritage to a space for nature, the city, and the people at the Jeonnam Textile and Ilshin Textile factory sites, which are symbols of industrialization in Gwangju and Jeollanam-do. The contents of the study are as follows. First, the concept and elements of the place and the placeness are identified as a theoretical background. Second, the properties and the characteristics of the boundary space as a medium to establish the relationship between the areas are figured out. Third, an architectural plan as an alternative to sustainable urban space revitalization is proposed. The contents of the architectural plan proposed in this study are summarized as follows. First, in order to redefine the relationship between the city and the nature around the proposed site, re-establish the boundary as a place where interaction can take place. Second, at the re-established boundary between nature and city, plan the frame element as an interact boundary combined with the existing industrial heritage, which plays a functional role of vertical and horizontal circulation naturally connecting nature and the city. Third, a transition space using existing buildings is planned around the railway that carried coal in the past and the road that crosses the site, which reveals the previously invisible boundary and expands two different areas. The existing buildings, which contain new programs by transforming the various physical boundaries that make up the space, can restore the historical and cultural value of the proposed site as a modern industrial heritage and return it to a space for people. The architectural plan proposed in this study is intended to provide alternatives to urban space revitalization that can restore the modern industrial heritage to a space for nature, the city, and the people.
\end{abstract}

Keywords: Urban Regeneration, Placeness, Boundary, Restoration, Interaction

\section{Introduction}

\subsection{Background and Purpose of the Study}

The cultural heritage related to industry left in the process of modernization in cities is called modern industrial heritage. In a broad sense, industrial heritage belongs to Brown Fields, which refers to land abandoned after a decline in function[1]. Numerous modern industrial heritages created through the process of industrialization have a value that can illuminate a series of historical flows such as politics, economy, society, and culture at the time[2]. Modern society recognizes the value of

Received: September 2, 2021; $1^{\text {st }}$ Review Result: October 18, 2021; $2^{\text {nd }}$ Review Result: December 4, 2021 Accepted: January 29, 2022 
modern industrial heritage that remains as idle spaces in the city and promotes urban regeneration through recycling, looking at it from a new perspective rather than urban aggression. Since the scope of modern industrial heritage is very wide, it is necessary to look at its recycling in connection with the society, economy, and culture of the time, rather than simply staying on the value of the heritage itself[3]. People remember places as traces of their memories. An important factor in the revitalization of urban space is the place value of space. Therefore, for sustainable urban regeneration, it is necessary to revitalize space by reviving the value of the place for the continuous flow of time, place, and events[4]. The modern industrial heritage is a place with historical significance that played an important role in the development of the city at that time. Therefore, the proposal of a reuse method of an industrial heritage can play an essential role as a method of sustainable urban revitalization that maintains the identity of the city and creatively converges the past and the present.

This study attempts to preserve the historical and cultural values of the modern industrial heritage and to find a way to interact with the modern industrial heritage, which has been cut off from the city. For this purpose, this study uses the Jeonnam Textile and Ilshin Textile factory sites, which are symbols of industrialization in Gwangju and Jeollanam-do, which have stopped operating, as proposed sites to restore the identity of the places that have gradually disappeared over time, and to propose an architectural plan as an alternative to sustainable urban space revitalization that can restore the modern industrial heritage to a space for nature, the city, and the people.

\subsection{Content of the Study}

The purpose of this study is to propose a plan for urban regeneration as an alternative in restoring the modern industrial heritage to a space for nature, the city, and the people through the restoration of the value as a place. The contents of the study are as follows. First, the concept and elements of the place and the placeness are identified as a theoretical background. Second, the properties of the boundary space and the characteristics of the boundary space as a medium to establish the relationship between the areas are figured out. Third, as an alternative for modern industrial heritage and urban space revitalization, an architectural plan based on the concept of the restoration and the interact boundaries are proposed.

\section{Theoretical Backgrounds}

The value of industrial heritage includes its intrinsic value and the value of a place that is revealed by its relationship with the surrounding environment. In particular, since an important element of urban revitalization is the place value of the space, this chapter attempts to understand the concept of the place and the placeness as theoretical backgrounds of this study, as well as the properties of the boundary and its characteristics.

\subsection{The Concept of the Place and the Placeness}

A place is a phase in which a naturally endowed environment is cultured. This means that the natural environment itself has the ability to generate as a place, so it is a good place for residence or gathering. A place has three semantic characteristics: a physical characteristic, an activity, and a symbol[5]. Placeness refers to the state in which a place is given meaning. In other words, the place is not an empty space, but is given meaning by humans to reveal the sense of the placeness. The placeness constituted around a place is a perceived characteristic of a place, and it refers to the characteristic that humans feel attached to by experience and is unique to one place and different from other places at the same time[6]. Therefore, it can be said that the sense of the placeness is the 
collective and social consciousness that members of a specific society have about the place that is their living base in the process of living together.

\subsection{Elements of the Sense of the Placeness}

The transition from space to a place can be explained by the relation of transition from space as object to space as perception and experience. Therefore, in the concept of the placeness, recognition through human perceptual experience can be identified as an important characteristic. Based on these characteristics, it can be understood that the sense of the placeness is created by combining the physical environment to construct a space, human activities in it, and the meaning of time and memory of the place. In architecture, the elements for forming the sense of the placeness include physical elements of the site and surrounding circumstances, integration with natural and cultural elements, differentiation from other places, connection with historical elements, and meaning creation according to human actions[6]. Specific elements of physical and environmental factors corresponding to the formation factors of a place are the natural environment and numerical location, the built environment made by human, the transportation system and infrastructure, and historical relics[7].

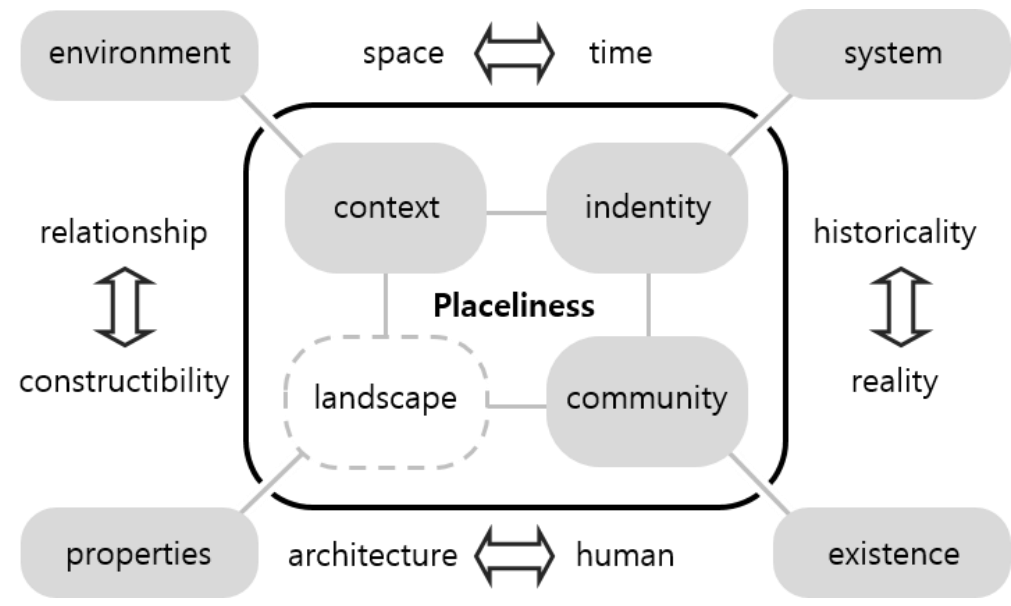

[Fig. 1] Elements of the Sense of Placeness[8]

According to the model in [Fig. 1], the placeness in Korean architectural discourse is surrounded by the concepts of context, identity, landscape, and community, and in a broader sense, it is also connected with the concepts of environment, system, properties, and existence. Each element of the sense of the placeness is divided by oppositions such as relationship-constructability, historicityreality, space-time, and architecture-human, but the sense of the placeness presented by their integration occurs at the interface of the opposites[8]. Thus, the concept of placeness arises and is reinforced between opposing relationships, and in this point of view, the nature of the boundary between opposing elements plays an important role in the formation of the sense of the placeness.

\subsection{Characteristics of Boundary in Modern City}

Space is perceived by its boundaries. In a space, boundaries of space are what defines the space, reflects the nature of the space, and appears as a result of the relationship between different spaces. The boundary not only blocks the inside and outside, but also adjusts the relationship through transformation. A regulation by the boundary affects human behavior in space. In a space, boundaries are integrated and separated by structural relationships including elements and relationships, and regulate human-space and human-human relationships[9]. By dividing a city that is perceived as a 
single organization into individual organizations, they are cut off by boundaries of various types, and in proportion to the diversity of the elements constituting the boundaries, the shape of the boundaries also varies[10]. Most of the boundaries are physically distinct and show strong disconnection. The natural environment of forests, rivers and water elements forms a boundary that divides regions in urban space. The fortress that used to form the outskirts of the city and the railways, roads, parks, and artificial walls created after modern times are artificially formed boundaries. Most of the elements of the boundary not only cut off exchanges with other areas by limiting a specific area in modern cities, but also act as a negative factor that hinders the composition of urban space. The boundary of these negative factors is an object that must be overcome for the restoration of urban order[11].

A boundary in a city is not only a line or a limit that divides the scope of a region, but also serves as a role of establishing relationships with things that exist on both sides of the boundary. In other words, in the process of city formation, boundaries have been operated to maintain the independence of each area, and this has resulted in the establishment of urban order. A boundary in a city should not simply separate two regions, but should be recognized as multiple interspaces with the character of a medium that establishes the relationship between regions. Thus, the boundary in urban space is an indeterminate place that occurs at the point where two different situations collide. In this sense, a boundary is a fence that indicates the territorial limits of objects, and at the same time plays a role of interaction between two different objects[12].

In modern architecture, the most basic principle of boundary demolition is to constantly create new relationships and create new interaction based on them[13]. In this way, boundaries in modern space play a role as a mediating area that allows space to interact with each other. Therefore, it can be said that the boundary is a transition from the role of confrontation and separation between areas to the relational role between areas. In space, the boundary is the part where transformation begins, where mutual exchanges and differences occur, and through this, mutual identities are generated. Due to the non-boundary nature of space, the territoriality between spaces is dismantled, simultaneity is secured, and the relationship in space is transformed from an antagonistic relationship to a complementary relationship[14].

This study focused on the characteristics of the sense of the placeness, which arises and strengthens between opposing relationships as a concept of a plan to restore the value of a place as a modern industrial heritage. Therefore, the direction of the plan was set so that the boundary space that sets the area of the place can play the role of interaction as an intermediary space.

\section{Architecture Plan for Modern Industrial Heritage and Urban Space Revitalization}

This study proposes an alternative for the revitalization of modern industrial heritage and urban space through restoration of value as a place. To this purpose, this study propose an architectural plan based on the concept of the restoration and interact boundary at the Jeonnam Textile and Ilshin Textile factory sites, which are symbols of industrialization in Gwangju and Jeonnam, both of which have stopped operating at present.

\subsection{Proposed Site}

The proposed sites of this study are the factory sites of the Jeonnam Textile and Ilshin Textile Factory located in Im-dong, Gwangju, a modern industrial heritage that has ceased operation. One of the sites is in contact with the nature of Gwangju Stream, and the other three are in contact with the residential area of the city, but there is hardly any connection inside the site. The surrounding roads are also in a state of being cut off for a long time due to the factory site that has stopped operating. 


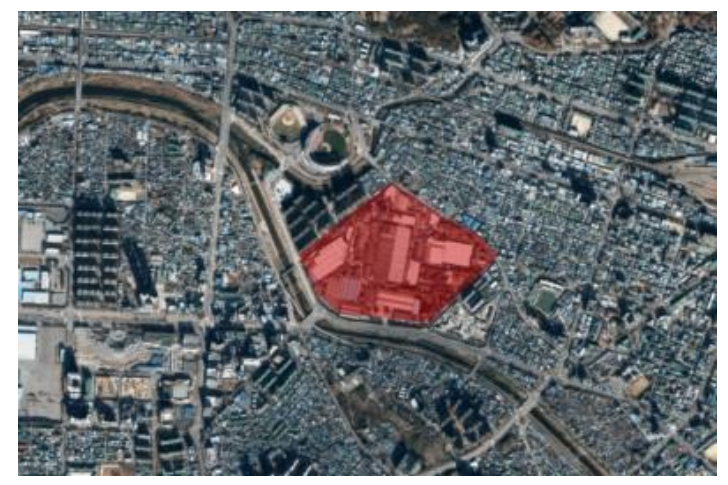

[Fig. 2] Proposed Site

\subsection{Restoration of Nature and Connection with the City}

In modern cities, the relationship between nature and the city has undergone many changes. The city, which used to create boundaries for protection from nature, gradually expanded its scope and formed a new boundary outside the boundary of nature. Due to urban development, it is increasingly difficult to find nature around the city, and the city and nature are gradually separated. This study avoids the modern city in which nature and city are separated, and returns to the coexistence of nature and city to restore the same relationship as before. This study suggests that in the future, nature and the city should appear in the form of a relationship that can be connected from an equal position, rather than as belonging to each other.
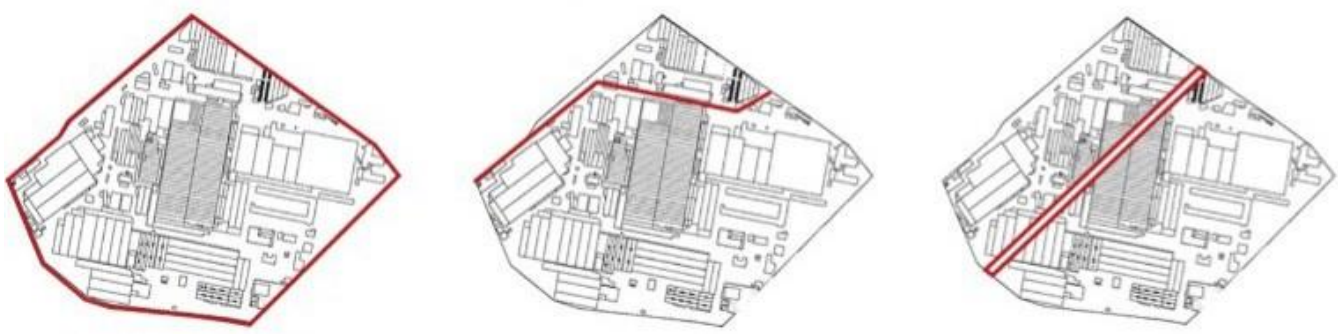

[Fig. 3] Boundaries Existing in Proposed Site

[Fig. 3] shows the boundaries of the proposed site. These boundaries have become a negative factor that cuts off relations with the city after the site ceased to function as an industrial facility. This study proposes to re-establish the boundary as a place where interaction can be made, not the disconnected boundary, in order to re-establish the relationship between the surrounding nature and the city of the proposed site where the relationship is currently cut off.

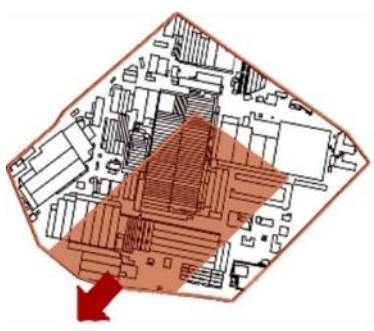

Reverting to nature

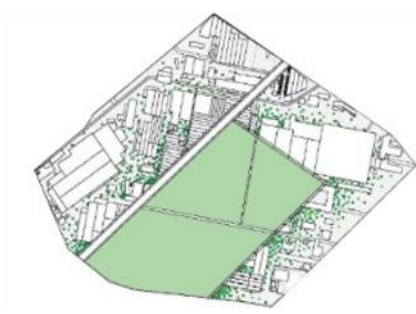

Regeneration plan

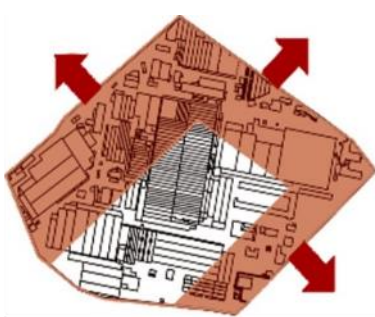

Connection to the city

[Fig. 4] Restoration to Nature and Connection with the City 
Among the two areas divided by the newly set boundary, the part adjacent to the Gwangju Stream is restored to nature, and the remaining areas adjacent to the city are newly connected with the city as shown in [Fig. 4]. The new boundary established between the city and nature makes it possible to serve as an interact boundary or a place that both the city and nature can interact.

\subsection{Architectural Plan Based on the Concept of Interact Boundaries}

\subsubsection{Reconfigured Boundary}

Restoration has a meaning of radically changing miscellaneous objects or phenomena, that is, returning to the original state. The concept of the restoration in this study means returning space, and it is a concept for sustainable urban regeneration that restores the sense of the place of the modern industrial heritage that exists cut off from the surroundings and connects nature and the city. For this purpose, this study proposes an alternative to reconfigure the boundaries of the proposed site. By reconfiguring the boundary, the transformation of space is made, and the closed and arbitrarily existing space is restored to nature and the city, acting as a space serving as a medium to connect the city. Reconfiguring the boundary is a proposal to break down the disconnected boundary and form an interact boundary.
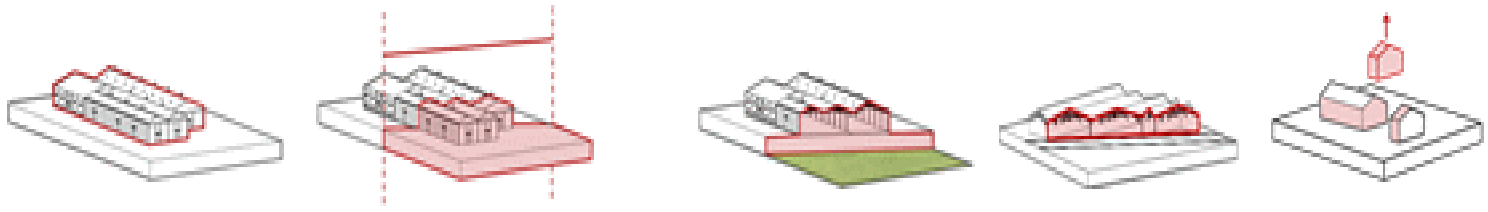

[Fig. 5] Change of the Shape of Space through Reconfiguration of Boundaries

In order to reconfigure the boundary, a new boundary should be set or an existing boundary should be modified to give a new function[Fig. 5]. A new boundary establishes a new relationship between spaces. An area is formed between spaces of different characteristics, and the area plays a role in naturally connecting the two spaces. The plan proposed in this study is a proposal to combine and transform various architectural devices according to the shape of the boundary so that the place of the boundary can play a role as a place of interaction. The method to form an interact boundary proposed in this study is as follows.
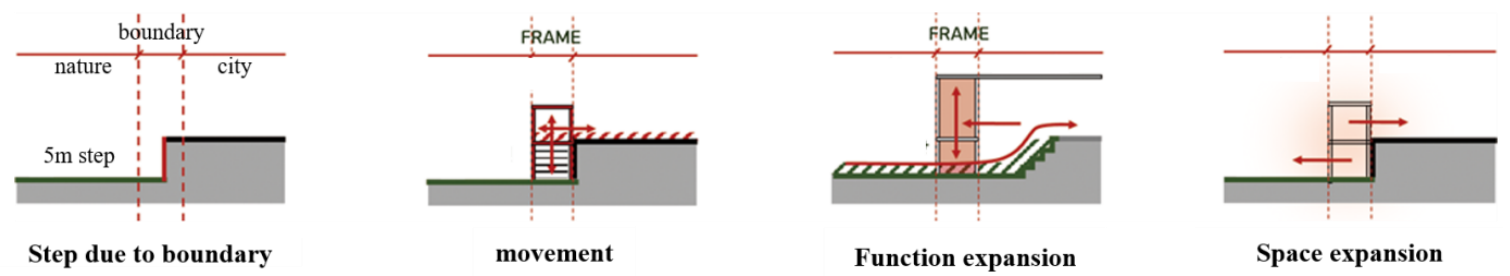

[Fig. 6] Interact Boundaries: Functions of the Frames

First, in the case of a boundary with a difference in height, the elements of the frame are applied to create an interact boundary as shown in [Fig. 6]. This frame is a device that can contain various functions within a frame with an open boundary. If the existing closed boundary brings about disconnection, the open boundary itself becomes a passage that provides extension to various functions. This frame space is a device that can overcome the spatial difference of different levels, and can enable natural spatial exchange between the two areas. 


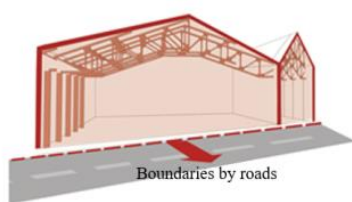

Boundary

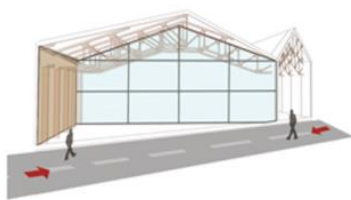

Elevation

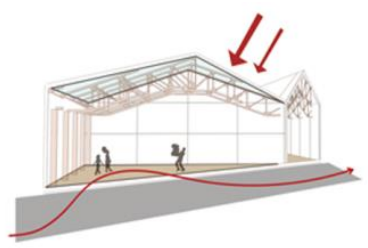

stay

[Fig. 7] Interact Boundaries: Functions of the Transition Space

Second, in the case of a boundary without a difference in height, an interact boundary can be formed in the form of a transition space as shown in [Fig. 7]. The new interact boundary has the purpose of connecting cities and cities, cities and nature, and nature and nature. Even if it is a space of the same nature, if the space is expanded by setting a new boundary, a transition space is created between the two areas, and the interact boundary between the two areas is established through this space.

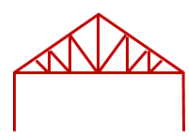

Structure

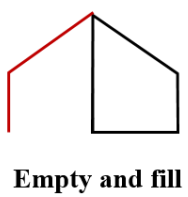

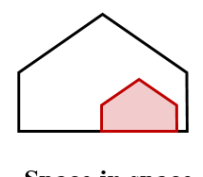

Space in space

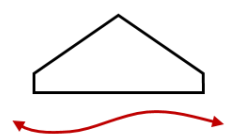

Filotti

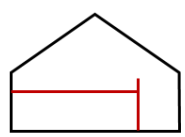

Wall and slab

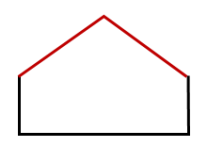

Roof

[Fig. 8] Interact Boundaries: Securing the Diversity of Space

Third, transform the various physical boundaries that make up the space as shown in [Fig. 8]. This is a method of planning that allows the boundary space to play a role as a place of interaction by changing the physical elements of the building, such as floors, walls, and roofs, to blur the boundaries between the inside and outside. This study proposes a plan that can secure the diversity of space, such as, removing the outer wall, to leave the structure, tearing down the walls and roof, adding a new space in the space, creating a piloti, dividing the space by adding a floor, and changing the material of the outer wall.

\subsubsection{Plans of Interact Boundary}

At the boundary between nature and city proposed in this study, a frame element combined with the existing industrial heritage is planned[Fig. 9]. This frame element plays a functional role of vertical and horizontal circulation, and plays a role in connecting nature and the city. The level difference between nature and urban space is overcome by the vertical circulation planned in the frame, and a natural approach to each area is induced so that the relationship between the city and nature can be close. In addition, the new area established between nature and the city by the frame has the possibility of expanding nature and urban space. When nature and the city are expanded like this, the boundary between nature and the city is gradually blurred and an interact boundary can be formed.
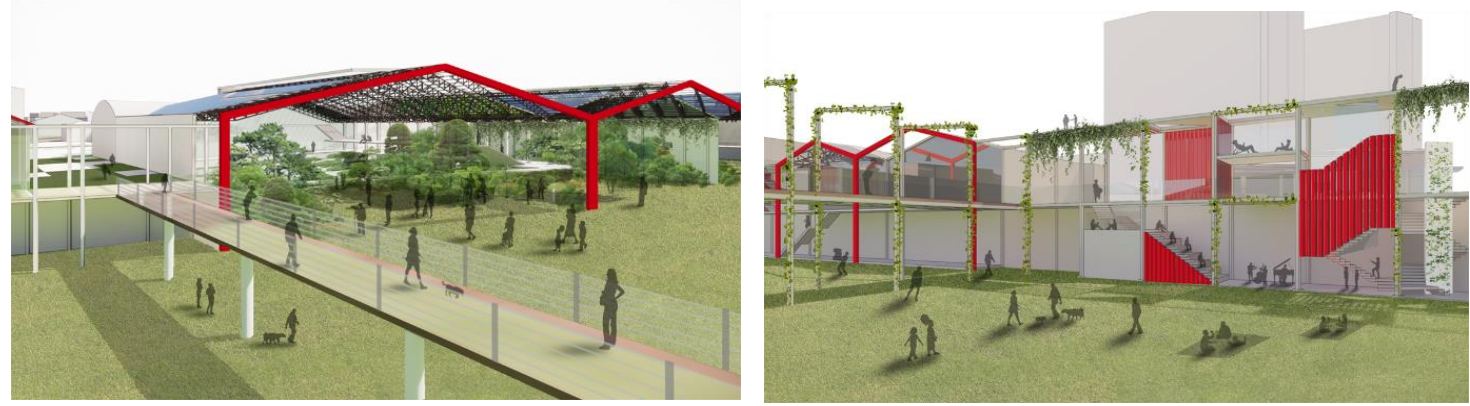

[Fig. 9] Proposal of the Interact Boundary between the Expanded Nature and the City 
In the area adjacent to the city within the proposed site, there is a railway that carried coal in the past and a road that crosses the site. In this plan, this is planned as a place that connects the inside and outside of the site as shown in [Fig.10]. Between this place and the remaining industrial heritage, a transition space using existing buildings is planned so that various activities of people can occur. This transition space reveals the previously invisible boundary and expands two different areas.
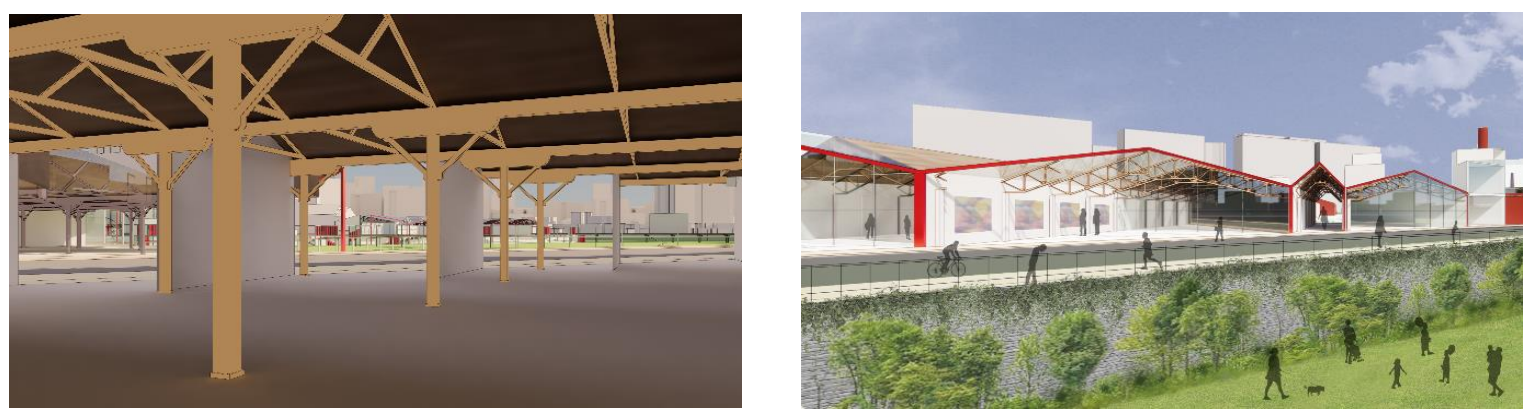

[Fig. 10] Proposal of the Interact Boundary as a Transition Space

The existing buildings as industrial heritages are planned as a place to contain new programs by transforming the various physical boundaries that make up the space[Fig. 11]. This place with a new program is a proposal to restore the historical and cultural value of the research site as a modern industrial heritage and return it to a space for people.
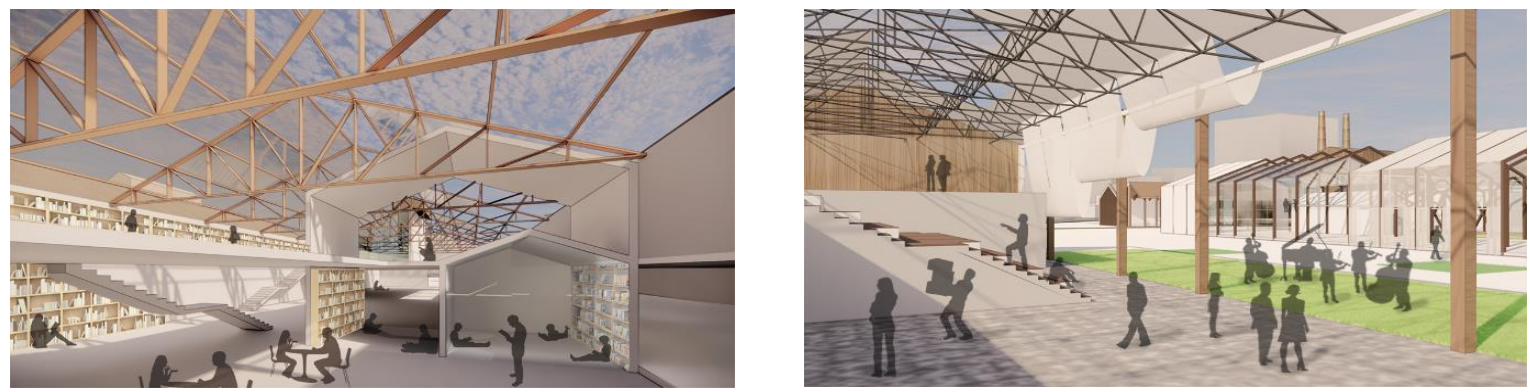

[Fig. 11] A Place for New Programs by the Transformation of the Physical Boundary of Buildings

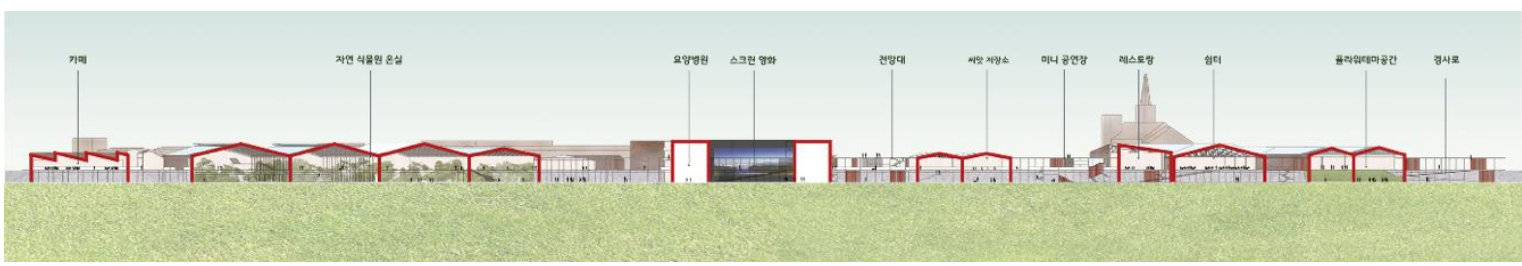

[Fig. 12] Architectural Program of Interact Boundary - East Side

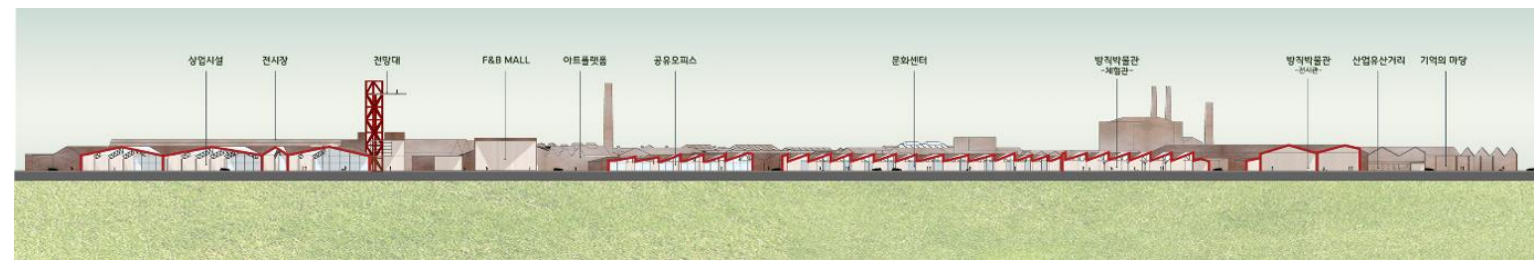

[Fig. 13] Architectural Program of Interact Boundary - West Side 


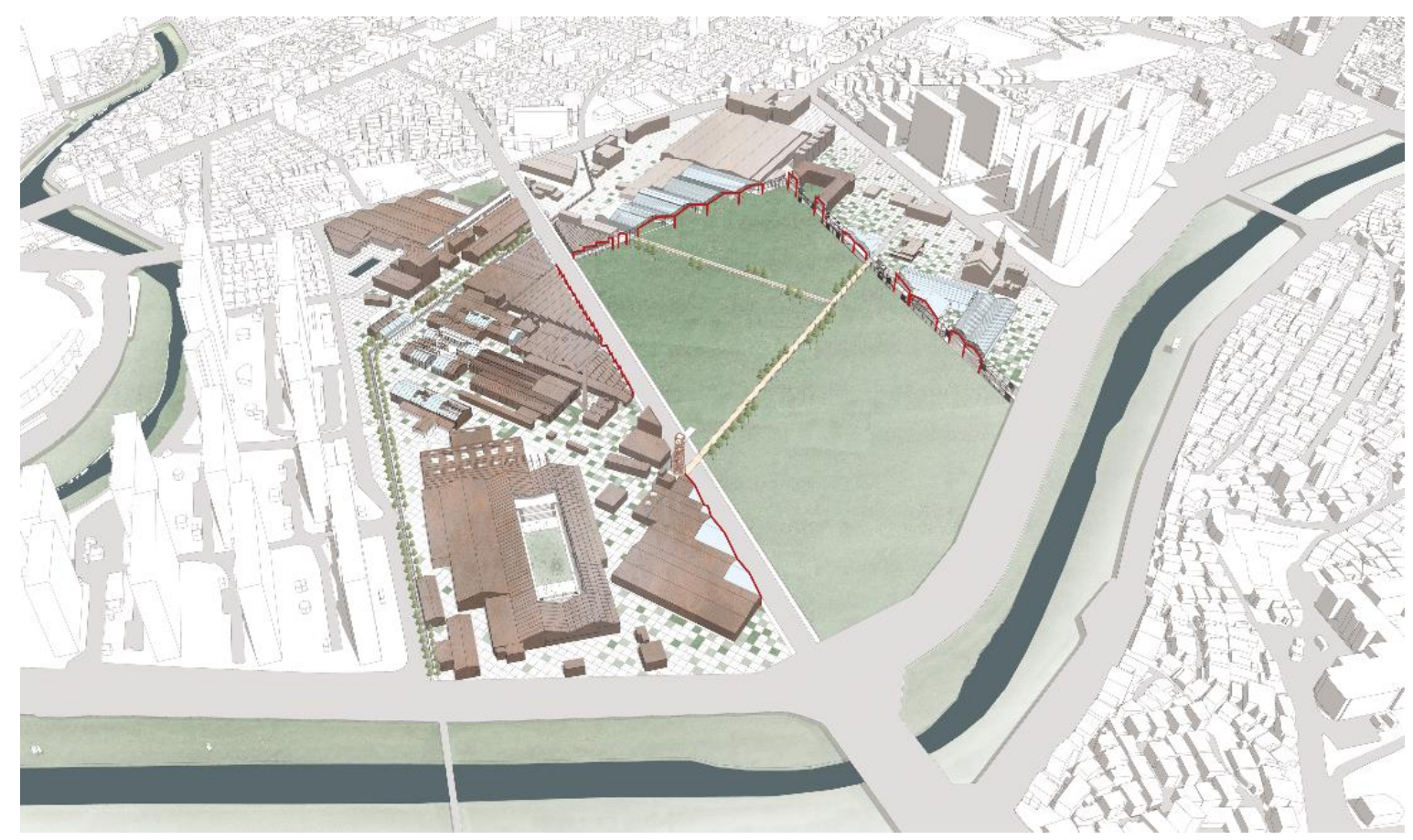

[Fig. 14] Architecture Plan for Modern Industrial Heritage and Urban Space Revitalization

\section{Conclusions}

This study attempted to preserve the historical and cultural values of the modern industrial heritage and found a way to interact with the modern industrial heritage and the city, which has been cut off from the city. This study proposed an architectural plan as an alternative to sustainable urban space revitalization that can restore the modern industrial heritage to a space for nature, the city, and the people at the Jeonnam Textile and Ilshin Textile factory sites, which are symbols of industrialization in Gwangju and Jeollanam-do. The contents of the architectural plan proposed in this study are summarized as follows. First, in order to re-establish the relationship between the city and nature around the proposed site where the current relationship is cut off, this study proposes to plans to restore the boundary as a place where interaction can be made, not the existing disconnected boundary. Second, at the boundary between nature and city proposed in this study, a frame element combined with the existing industrial heritage is planned. This frame element plays a functional role in vertical and horizontal circulation, and in connecting nature and the city. Third, a transition space using existing buildings is planned around the railway that carried coal in the past and the road that crosses the site. This transition space reveals the previously invisible boundary and expands two different areas. The existing buildings as industrial heritages are planned as a place to contain new programs by transforming the various physical boundaries that make up the space. This place with a new program is a proposal to restore the historical and cultural value of the proposed site as a modern industrial heritage and return it to a space for people. The architectural plan proposed in this study is an alternative to urban space revitalization that can restore the modern industrial heritage to a space for nature, the city, and the people. However, this study focuses only on the change of the physical environment of the proposed site, thus there is an insufficiency of proposals for specific programs for various uses that match the characteristics of existing buildings. Therefore, follow-up studies of creative programs that can contribute to regional revitalization as a method of sustainable urban revitalization that maintains the identity of the city and converges the past and the present are needed. 


\section{References}

[1] M. J. Jeong, A Study on the Recycling Tendency of Domestic Abandoned Factories, KIID Autumn Conference Proceeding, Korea Institute of Interior Design, pp.86-90, (2013), November 9, Seoul, Korea.

[2] D. J. Kang, S. K. Oh, Exploring of the Industrial Heritage's Reuse Methods: With Focus on Japan Precedents, Journal of The Urban Design Insitute of Korea, (2003), Vol.12, No.3, pp.59-71, UCI: G704-001620.2003.12.3.001

[3] D. J. Kang, S. H. Lee, D. S. Choi, An Analysis of Concept and Conservation Methods for Industrial Heritage, Journal of Korea Planning Association, (2003), Vol.38, No.2, pp.7-20, UCI: G704000338.2003 .38 .2 .015

[4] M. T. Lim, J. H. Yoo, A Study on the Application of Pop-Up Concept for Urban Space Revitalization, Journal of Korea Intitute of Spatial Design, (2014), Vol.9, No.4, pp.163-174, DOI: https://dx.doi.org/10.35216/kisd.2014.9.4.163.

[5] S. H. Lee, K. W. Hwang, The Ambiguous Concepts of Place and Placeness, Journal of Korea Planning Association, (1997), Vol.32, No.5, pp.169-184.

[6] D. H. Choi, A Study of the Architectural Planning Based on the Concept of Sense of Place -The Cultural Complex at the Thames River-, Journal of the Korean Society of Science and Technology, (2016), Vol.17, No.11, pp.513-520, DOI: http://dx.doi.org/10.5762/KAIS.2016.17.11.513

[7] J. Y. Uhr, H. K. Yuh, A Study on Place Value on the Notion Place, Journal of Korea Planning Association, (2010), Vol.45, No.6, pp.21-34, UCI: G704-000338.2010.45.6.002

[8] I. S. Kim, A Study on the Diachronic and Synchronic Structure of the Concept of Place in Korean Architecture Focused on the Korean Architectural Discourse during Around 1990s-, Korean Institute of Interior Design Journal, (2015), Vol.24, No.1, pp.124-132, DOI: http://dx.doi.org/10.14774/JKIID.2015.24.1.124

[9] S. W. Hong, K. H. Lee, Y. S. Kim, A Study on the Ambiguity of Architectural Space by the Expended Boundary, Proceeding of Annual Conference of the Architectural Institute of Korea Planning \& Design, Korea Institute of Interior Design, pp.176-179, (2005), October 24-25, Seoul, Korea.

[10] Y. J. Cho, A study on the architectural resuscitation of the in-between space in the urban bloc, Konkuk University Graduate School of Architecture, Master's thesis, pp.8-10, (2003)

[11] I. S. Son, H. J. Yoo, Space-Forming of the Border for Understanding the Urban Space - Focus on the Disconnected Border of the Wall, Proceeding of Annual Conference of the Architectural Institute of Korea Planning \& Design, Architectural Institute of Korea, pp.161-164, (2007), October 26-27, Cheongju, Korea.

[12] J. H. An, Y. M. Koo, A Study on an Acquisition of Public Sphere at Urban Border through Landscape Architecture, Proceeding of Annual Conference of the Architectural Institute of Korea Planning \& Design, Architectural Institute of Korea, pp.97-98, (2010), October 23, Cheongju, Korea.

[13] S. M. Park, Y. S. Lee, A study on the Characteristics of the Blurred Zone in Contemporary Architecture's Boundaries, Proceeding of Autumn Annual Conference of the Architectural Institute of Korea Planning \& Design, Architectural Institute of Korea, pp.269-272, (2011), October 29, Gyeongsan, Korea.

[14] H. J. Lee, S. G. Oh, A Study on the Expression of the Contemporary Architecture By Cognition of Boundary, Proceeding of Autumn Annual Conference of the Architectural Institute of Korea Planning \& Design, Architectural Institute of Korea, pp.311-314, (2003), October 24-25, Busan, Korea. 